\title{
IMPROVING FREEWAY SPEEd ESTIMATES FROM SINGLE-LOOP DETECTORS
}

\author{
Bruce R. Hellinga 1 P.E.
}

\section{Abstract:}

Many existing freeway traffic management systems (FTMS) use speed data from loop detectors as input to various traffic management functions, such as automatic incident detection, and to traveler information system components. In many cases, these FTMS include single-loop detectors, which are not able to measure vehicle speed. Typically, speed estimates are made on the basis of single-loop traffic volume and occupancy measurements and estimates of average vehicle length. Unfortunately, the accuracy of these speed estimates is generally very poor. This paper presents a method for improving these speed estimates. The proposed method is applicable to FTMS that contain both single and dual-loop detector stations. It does not require modification to field hardware and does not require additional field equipment. The proposed method reduces the root mean squared speed estimation error by $23 \%$ on average over the traditional speed estimation method of using a constant average effective vehicle length for the entire day.

\section{Introduction:}

Many large urban centers in North America, Europe and Asia experience freeway congestion for significant portions of the day. As a result of pressure to manage and mitigate congestion, many

\footnotetext{
${ }^{1}$ Assistant Professor, Department of Civil Engineering, University of Waterloo, Waterloo, Canada

Phone: 519 888-4567 Ext. 2630; Fax: 519 888-6197; Email: bhellinga@uwaterloo.ca
}

Key Words: freeway traffic, speed estimates, loop detectors 
jurisdictions have implemented freeway traffic management systems (FTMS). These FTMS have typically obtained traffic data from induction loop detectors embedded in the roadway, that provide volume, occupancy, and in the case of dual-loop detectors, speed data. While recent technological developments have resulted in the availability of a wide range of traffic surveillance systems, including video imaging, radar, and dedicated short range communication, most FTMS have legacy infrastructure systems that include both single and dual-loop detector systems.

Travel speed is one of the primary traffic measures used for congestion management and providing information to drivers. While dual-loop detectors are able to measure vehicle speed directly, single-loop detectors can not. Many FTMS still have single-loop detector stations within their systems, and attempt to estimate speed from the measured traffic volume and occupancy, and assumptions about average vehicle length. Unfortunately, these speed estimates tend to be quite inaccurate.

This paper examines the problem of estimating speeds from single-loop detectors and proposes an algorithm, applicable to FTMS that contain both single and dual-loop detectors, that can be used to improve the accuracy of speed estimates from single-loop detectors. The algorithm does not require any additional field equipment or any modifications to existing loop configurations. Implementation of the proposed algorithm only requires minor modifications to the software in the traffic management center (TMC) used to process the loop detector data.

\section{Background}

As illustrated in Figure 1, dual-loop detectors are able to directly measure vehicle speeds by tracking the time required for the vehicle to travel from the upstream loop detector to the downstream loop detector $\left(t_{2}-t_{1}\right)$. The separation distance between the two loop detectors $(D)$ is constant and is known, and thus the speed of the $j^{\text {th }}$ vehicle in polling interval $i\left(s_{i j}\right)$ is simply computed as the distance $D$ divided by the travel time $\left(t_{2}-t_{1}\right)$. The average space-mean speed, or harmonic mean, for 
vehicles passing over the detector during polling interval $i$, is computed as the distance, $D$, divided by the mean time for the observed vehicles to traverse the two detectors (Equation 1).

$$
\bar{S}_{i}=\frac{D}{\frac{1}{n_{i}} \sum_{j=1}^{n_{i}}\left(t_{2, j}-t_{1, j}\right)}=\frac{1}{\frac{1}{n_{i}} \sum_{j=1}^{n_{i}}\left(\frac{1}{S_{i j}}\right)}
$$

Detector occupancy is computed as the proportion of the polling interval duration that a vehicle is detected by the loop detector. As illustrated in Figure 2, occupancy is dependent on the effective detection zone length of the loop detector, the length of the vehicle, the speed of the vehicle, and the polling interval duration. In practice, it is difficult to separate the length of the $j^{\text {th }}$ vehicle in polling interval $i\left(L_{v, i j}\right)$ from the effective detection zone length $\left(L_{d}\right)$. Therefore, we define the effective length of the $j^{\text {th }}$ vehicle in polling interval $i\left(L_{i j}\right)$ as the sum of $L_{d}$ and $L_{v, i j}$ (Equation 2).

$$
O_{i}=\frac{1}{T} \sum_{j=1}^{n_{i}}\left(\frac{L_{v, i j}+L_{d}}{s_{i j}}\right)=\frac{1}{T} \sum_{j=1}^{n_{i}}\left(\frac{L_{i j}}{s_{i j}}\right)
$$

The average effective vehicle length in polling interval $i$ is given by,

$$
\bar{L}_{i}=\frac{1}{n_{i}} \sum_{j=1}^{n_{i}}\left(L_{i j}\right)
$$

For convenience, we define $V_{i}$ as the equivalent hourly traffic volume observed during polling interval $j$ (Equation 4).

$$
V_{i}=\frac{n_{i}}{T}
$$

Then substituting Equation 4 into Equation 2 provides,

$$
O_{i}=V_{i} \frac{1}{n_{i}} \sum_{j=1}^{n_{i}}\left(\frac{L_{i j}}{s_{i j}}\right)
$$

Then, if we assume that the effective lengths of all vehicles observed during polling interval $i$ are equal (i.e. $\bar{L}_{i}=L_{i j}, j=1,2,3 \ldots n_{i}$ ), and make use of Equation 1, we can obtain Equation 6. 


$$
O_{i}=V_{i} \bar{L}_{i} \frac{1}{n_{i}} \sum_{j=1}^{n_{i}}\left(\frac{1}{S_{i j}}\right)=\frac{V_{i} \bar{L}_{i}}{\bar{S}_{i}}
$$

Therefore, the average space-mean-speed can be estimated from detector occupancy, volume, and average effective vehicle length (Equation 7).

$$
\bar{S}_{i}=\frac{V_{i} \bar{L}_{i}}{O_{i}}
$$

The assumption that all vehicles in polling interval $i$ have the same effective length is frequently invalid. Furthermore, the use of Equation 7 requires knowledge of the detector volume and occupancy, and the average effective vehicle length for polling interval $i$. Single loop detectors are able to measure volume and occupancy, but are not able to provide average vehicle length. The most common approach adopted by transportation agencies is to assume some average vehicle length and use this constant value for all polling intervals during the day. Another method that is used in systems having both single and dual-loop detector stations, is to use the average vehicle length computed at a dual-loop station for estimating average speed at a nearby single-loop station. Neither of these two methods has proven to provide satisfactorily accurate speed estimates.

In the next section we examine several methods that have been proposed by other researchers. We then describe the field data used to test the methods proposed in this paper. The proposed methods are described and evaluation results are presented and discussed. Finally, conclusions are made regarding the value of the proposed methods.

\section{Existing Approaches}

Several other researchers have also examined the problem of estimating speeds from single loop detectors. Hall and Persaud (1989) and later Pushkar et al (1994) examined the validity of Equation 7 (albeit in a different form). They formulated an expression for the average space-mean- 
speed in polling interval $i$ on the basis of the fundamental speed-flow-density relationship (Equation 8) and by assuming density and occupancy were linearly related (Equation 9).

$$
\begin{gathered}
V_{i}=\bar{S}_{i} K_{i} \\
K_{i}=\frac{O_{i}}{\bar{L}_{i}}
\end{gathered}
$$

Substituting Equation 9 into 8 provides,

$$
\bar{S}_{i}=\frac{V_{i} \bar{L}_{i}}{O_{i}}
$$

which is the same expression for occupancy as Equation 7.

Hall and Persaud define $g_{i}$ as a speed estimation parameter for polling interval $i$ that is inversely proportional to the average effective vehicle length of interval $i$ (Equation 11). Thus the final form of the equation used by Hall and Persaud is provided in Equation 12.

$$
\begin{gathered}
g_{i}=\frac{1}{\bar{L}_{i}} \\
\bar{S}_{i}=\frac{V_{i}}{O_{i} g_{i}}
\end{gathered}
$$

Hall and Persaud concluded that Equation 12 is not strictly valid as Equation 8 is only true for uniform traffic flow and Equation 9 is only true when vehicle speeds and lengths are constant. These conclusions are not unexpected, as we have already seen in the derivation of Equation 7 that it is necessary to assume that effective vehicle lengths in interval $i$ are uniform. On the basis of field data obtained from truck restricted lanes, Hall and Persaud concluded that $g$ is not a constant, and therefore using a constant value for $g$ in Equation 12 (or a constant value for $\bar{L}_{i}$ in Equation 7) gives biased results.

More recently, research by Coifman (2000) suggests that $g_{i}$ is in fact constant, and that the variations in $g$ observed by Hall and Persaud result from the use of time-mean speed instead of space-mean speed and from using detector occupancies recorded in truncated integer format. 
In practice, when Equation 7 is implemented using a constant value for $\bar{L}_{i}$ (i.e. $\bar{L}_{i}=L, \forall i$ ), a value for $L$ is generally estimated by selecting single-loop detector volume and occupancy data from some period during the day when free-flow conditions are expected, assuming average speed, and using these values in Equation 7 to solve for $\bar{L}_{i}$. The constant average effective vehicle length $L$, computed as the average of $\bar{L}_{i}$ for the period when free-flow conditions are expected, would then be used in Equation 7 for the entire day.

Coifman (2000) suggests that improved estimates of speed can be obtained from Equation 7 if $L$ is estimated from all periods of the day in which free-flow conditions are observed, rather than just some pre-specified period of the day.

Wang and Nihan (2000) proved that knowledge of real-time average effective vehicle length during each polling interval is key for more accurate speed estimates using Equation 7. They opted to estimate mean effective vehicle length using a log-linear regression model that was calibrated using data from dual-loop stations. Their results indicated a $41 \%$ increase in the speed prediction accuracy when compared to using a constant value for $L$. However, they did not show that the regression model was transferable to other FTMS or even to other detector locations within the same FTMS.

Daily (1999) developed a speed estimation algorithm that considers individual vehicle speed and length to be random variables. The algorithm estimates speed on the basis of the statistical properties of the measured volume and occupancy, and the standard deviation of individual vehicle speeds. In practice, however, the standard deviation of individual vehicle speeds is not provided by loop detectors and therefore must be obtained through other means. The algorithm provides a reliability test for the speed estimate, however this test assumes that the distribution of vehicle lengths remains constant throughout the entire day. 
In this paper we examine methods for improving the speed estimates from Equation 7. These methods differ from those proposed by other researchers in that they are applicable to FTMS that have a mix of single and dual-loop detectors. In these mixed systems, average effective vehicle length can be directly obtained from dual-loop stations through the application of Equation 7 and solving for $\bar{L}_{i}$. It would be expected that using these measured effective vehicle lengths would improve the speed estimates for the single-loop stations.

The next section describes the field data used to test the proposed methods of reducing the error in the estimated speeds at the single-loop stations.

\section{Description of Field Data}

This study made use of loop detector data from Highway 401 in Toronto, Canada. This section of freeway experiences an AADT of approximately 320,000 vehicles, making it one of the most heavily traveled roadways in North America. The facility serves as the primary east-west commuting corridor for the City of Toronto as well as the primary east-west link for goods movements in the province of Ontario.

The COMPASS freeway traffic management system (Korpal, 1992; Binkley, 1999) provides surveillance via single and dual induction loop detectors and closed circuit television (CCTV) along approximately $45 \mathrm{~km}$ of the system. Loop detector stations are located at all access and egress points and approximately every $0.6 \mathrm{~km}$ along the freeway. The loop detectors provide volume, occupancy and in the case of dual-loop detectors, space-mean-speed data, to the COMPASS control center every 20 -seconds. Approximately $30 \%$ of the loop detectors are dual-loop stations, while the remainder are single-loop stations.

Detector data were obtained from 10 dual-loop stations located in the express and collector facilities of Highway 401 (Figure 3). For each detector station, 20-second speed, volume, and occupancy data were obtained for 5 weekdays in 1995 (October 9, 10, 11, 12, and 13). The 10 
selected detector stations were divided into 5 sets of stations (Table 1). For each set, one station was chosen to represent a single-loop station (for which a speed was to be estimated) and the other was treated as a dual-loop station and used to provide the average effective vehicle length. Since both stations of each set were actually dual-loop stations, an estimate of speed made for the "single-loop" station could be compared to the actual measured speed.

\section{Speed Estimation Methodologies}

This section defines the speed estimation methods that were evaluated using field data. The proposed methods can be divided into 4 categories; namely the Base Case, Direct Correlation, Filtered Correlation, and Bias Correction. Each of these methods is described below.

\section{Base Case}

We assume that the base case consists of estimating single-loop speeds on the basis of average effective vehicle length measured at a dual-loop station over the entire 24-hour period. This assumed base case is likely to provide speed estimates that are more accurate than would normally be obtained for FTMS with only single-loop detectors, for in these systems an average vehicle length must be assumed as it cannot be measured.

\section{Direct Correlation}

When some of the FTMS loop detectors are dual-loop stations, then it is possible to estimate average speed for each single-loop station on the basis of Equation 7 and the average effective vehicle length measured at a nearby dual-loop station during the same polling interval. This is the method used by the COMPASS system in Toronto.

Of course, when $\bar{L}_{i}$ is taken from a nearby dual-loop station for use in Equation 7, the implicit assumption is that the average effective vehicle length computed during the polling interval at the dual-loop station is highly correlated with the unknown average effective vehicle length at the 
single-loop station for the same time period. If average effective vehicle length at the single-loop station is not highly correlated with the average effective vehicle length at the dual -loop station, then additional error is introduced into the calculation of speed at the single-loop detector station.

\section{$\underline{\text { Filtered Correlation }}$}

As will be shown, the Direct correlation method does not perform well, primarily as a result of the lack of correlation between the average effective vehicle length at the single and dual-loop stations. For example, the correlation between the average vehicle lengths measured at the two detectors in detector station set 2 is only 0.085 . This lack of correlation is most likely a result of sampling error. During a short time interval (say 20-seconds), the average length of vehicles passing one detector station is likely to be different than the average length of vehicles passing a station at some other location because the vehicles passing each station represent different samples from the population of vehicles. The average length of vehicles passing a detector station during a polling interval is the result of a random sampling process in which the variation of the sample mean vehicle length is a function of the sample size and the variation of vehicle lengths within the population.

If it were assumed that the same population of vehicles passes each station, it would be expected that as the sample size increases, the differences between the sample means would decrease. Increasing the sample size can be achieved by computing the average effective vehicle length over a longer time period (i.e. using data from more than a single detector polling interval). If the population mean vehicle length is not constant, but varies with time of day, then averaging over a long period of time will result in estimates that do not adequately reflect these temporal trends.

If the same population of vehicles does not pass both detector stations (as is likely to be the case for at least some locations as a result of disproportionately higher truck volumes), then even when sample sizes are large, the sample mean at the dual-loop station will not approach the value of 
the population mean at the single-loop station. Instead, a systematic bias will exist in the speed estimates, which must be corrected through other means.

The difficulty that arises when selecting a longer time period over which to compute the sample mean vehicle length is the appropriate duration of the period. For a stationary process (i.e. one in which the mean does not change with time), as the period duration increases, the sample size increases, and the reliability of the sample mean increases. However, as illustrated in Figure 4, the vehicle length distribution has a strong temporal component as the proportion of long vehicles within the traffic stream varies throughout the day. For non-stationary processes, the appropriate sampling period duration depends on the characteristics of the population mean.

One way to avoid the problem of having to select a fixed sampling period duration is to use an exponentially weighted moving average (EWMA).

Exponential smoothing is an averaging technique that can be used when the appropriate averaging period duration is not known. The exponentially smoothed value is equivalent to a weighted average of all previous values and is computationally efficient as only two values need to be known at any time; the exponentially smoothed value from the previous time period, and the observed value from the current period.

Equation 13 can be used to compute an exponentially smoothed value at each detector polling interval $i$. This smoothed value can then be used in Equation 7 for estimating the average speed for the single-loop detector.

$$
Y_{i}=\left\{\begin{array}{cc}
X_{i} & i=1 \\
Y_{i-1} & i>1, X_{i}<0 \\
(1-\gamma) X_{i}+\gamma Y_{i-1} & \text { otherwise }
\end{array}\right.
$$

Each detector speed and occupancy measurement (i.e. $X_{i}$ from Equation 13) is actually an average computed from the number of vehicles (volume) passing the detector during the polling interval. Since volume varies temporally, it may be prudent to consider the volume within the 
exponential smoothing process. This may be accomplished by recognizing that the constant decay coefficient $\gamma$ of Equation 13, which is the weighting applied to the smoothed value from the previous time period, should vary depending on the number of vehicles that contribute to the observation of the current period. For example, if the current observation is based on 10 vehicles, it should have a higher weighting than if it is based on only 1 vehicle. In this case, a weighting coefficient $\alpha$, which is a function of a decay constant $\beta$ and the number of vehicles associated with the current observation, is applied each period (Equation 14). As volume increases, $\alpha_{i}$, the weight placed on the current observation $\left(X_{i}\right)$, increases and as volume decreases, $\alpha_{i}$ decreases. This effect is desirable, as the greatest variation in vehicle length appears to coincide with periods of the day with low traffic volume.

$$
\begin{gathered}
Y_{i}=\left\{\begin{array}{cc}
X_{i} & i=1 \\
Y_{i-1} & i>1, X_{i}<0 \\
\left(1-\alpha_{i}\right) X_{i}+\alpha_{i} Y_{i-1} & \text { otherwise }
\end{array}\right. \\
\text { where } \alpha_{i}=\beta^{n}
\end{gathered}
$$

\section{Bias Correction}

When the population of vehicles passing the dual-loop station has a different mean effective vehicle length than does the population passing the single-loop station, then a systematic bias exists. If both stations are dual-loop stations, than the presence of a systematic difference can be examined by comparing the average 24-hour effective vehicle length measured at the two stations. Of course, if both stations are dual-loop stations, then it is not necessary to estimate speed, as it can be directly measured. However, it is useful to consider the performance improvement that could be achieved if the average effective vehicle length at both stations can be determined, as this may help to explain the source of estimation errors for other methods. Systematic differences in effective average vehicle length are considered in the estimation of single-loop speeds by using Equation 15 to estimate $L^{\prime}$ and then using $L^{\prime}$ in Equation 7 instead of $\bar{L}_{i}$. 


$$
L^{\prime}=L+\left(L_{S}-L_{D}\right)
$$

In practice it is not possible to correct for differences in effective average vehicle lengths using Equation 15, as it is not possible to determine vehicle length at single-loop stations. However, it may still be possible to compensate, at least in part, for differences in detector station calibration and different vehicle populations, by considering systematic differences in average vehicle speed. In this case, average vehicle speed is computed at each detector over a time period during which near free-speed conditions are expected (e.g. 12 midnight to 5 AM). For the dual-loop station, the average of the measured vehicle speeds is computed. For the single-loop station, the average of the estimated speeds is computed. Unless specific geometric conditions exist that would cause an increase or decrease in average vehicle speeds, under near free-flow conditions, we would expect the average speeds at both detector locations to be the same. If a difference does exist, it is likely a result of a systematic bias in the detector calibration or differences in average vehicle lengths, and therefore should be considered in the speed estimation process. In this case, Equation 16 can be used to estimate the speed at single-loop stations.

$$
\bar{S}_{i}=\frac{V_{i} \bar{L}_{i}}{O_{i}}\left[\frac{\bar{S}_{D}^{5 h}}{\bar{S}^{5 h}}\right]
$$

\section{Evaluation}

The above methodologies can be considered as treatments applied to the various field data. Two treatments were applied to the single-loop detector volume and occupancy measurements; (1) raw 20-second data and (2) volume weighted EWMA (Equation 14). Four treatments were applied to the effective vehicle length computed from the dual-loop detector data; (1) 24-hour average, (2) raw 20-second length, (3) EWMA of 20-second length data (Equation 13), and (4) volume weighted EWMA of 20-second length data (Equation 14). Finally, three bias correction treatments were 
considered; (1) no correction, (2) theoretical correction (Equation 15), and (3) practical correction (Equation 16).

Speed estimation scenarios were created by selecting combinations of treatments applied to the single-loop volume and occupancy data, to the vehicle lengths obtained from the dual-loop detector, and by selecting a bias correction treatment. Table 2 identifies each of the 20 combinations of speed estimation methodologies that were examined. Scenario 1 represents a direct application of Equation 7 as typically used in FTMS and can be considered the base case. Scenario 2 represents the speed estimation method used by the Ontario Ministry of Transportation.

Speed estimation accuracy was quantified using the average root-mean-square error computed across all 5 weekdays and all 5 sets of detector stations (Equation 17).

$$
E_{n}=\frac{1}{25} \sum_{d=1}^{5}\left(\sum_{p=1}^{5}\left[\frac{1}{I} \sum_{i=1}^{I}\left(\bar{S}_{d, p, i}-\hat{S}_{d, p, i}\right)^{2}\right]^{0.5}\right)
$$

\section{Results}

Figure 5 illustrates the average root-mean-square speed estimation error for each of the 20 scenarios. The legend beneath the figure indicates which treatments have been applied to the singleloop data, the vehicle length computed from the dual-loop detector, and what bias correction has been applied.

Two observations can be made from these results.

First, the average RMSE $\left(E_{n}\right)$ ranges from $9.7 \mathrm{~km} / \mathrm{h}$ to $20.4 \mathrm{~km} / \mathrm{h}$, indicating that for all scenarios, the speed estimation error remains rather large.

Second, the base scenario, in which the 24-hour average vehicle length is used, does not provide the most accurate speed estimates.

To better appreciate the performance of the speed estimation methods in comparison to the base case (i.e. to typical existing practice), the performance of the speed estimation methods is 
compared to the base case by examining the percent improvement in speed estimation accuracy (Equation 18).

$$
\Delta_{n}=\frac{E_{\text {base }}-E_{n}}{E_{\text {base }}} \times 100 \%
$$

Figure 6 illustrates the performance of each speed estimation scenario compared to the base case. Several observations can be made from these results.

First, scenarios in which the speed estimation is made on the basis of the 20 -second vehicle length from the dual-loop station (scenarios 2, 6, 10, 14, and 18) consistently provide poor results. This is consistent with expectation, as there is very low correlation between the average vehicle length observed at the single-loop station and the average vehicle length observed at the dual-loop station in the same 20 -second interval.

Second the application of EWMA or volume weighted EWMA to dual-loop length alone (scenarios 3 and 4) does not provide substantial improvement over the base case. However, significant improvement (almost 20\%) is obtained when the volume weighted EWMA is also applied to the single-loop detector volume and occupancy measurements.

Third, the use of Equation 16 to correct for systematic biases in either detector calibration or different vehicle populations has only a small benefit (approximately 3\%) and only when used in conjunction with EWMA or volume weighted EWMA.

Fourth, the use of Equation 16 to correct for systematic biases captures only a small portion of the systematic bias error. For example, Scenario 15 provides a $35 \%$ improvement over the base case when the theoretical bias correction (Equation 15) is used. However, in practice, the use of Equation 16 (Scenario 11) only provides an improvement of $21 \%$.

Lastly, of all scenarios that can be implemented, Scenario 12 provides the greatest improvement over the base case (23\%). This implies that volume weighted EWMA should be applied to the 20-second single-loop volume and occupancy measurements and the 20 -second average 
vehicle lengths obtained from the dual-loop detectors, and that the speed correction should be applied.

The use of Equation 14 for volume weighted EWMA requires the selection of an appropriate value for the decay coefficient $\beta$. Optimal values for $\beta$ were found by exhaustive enumeration for each of the speed estimation scenarios using Equation 14. Figure 7 illustrates the sensitivity of the average $\operatorname{RMSE}\left(E_{n=12}\right)$ to the value of the decay coefficient $(\beta)$ from Equation 14 and applied to the effective vehicle length obtained from the dual-loop station for Scenario 12. Similarly, Figure 8 illustrates the estimation error $\left(E_{n=12}\right)$ as a function of the decay coefficient $\beta$ applied to the singleloop volume and occupancy data. The results in both of these figures indicate that the recommended estimation method is relatively insensitive to the value of $\beta$ over the range of $0.85 \leq \beta \leq 0.99$. This result is desirable as it seems to indicate that the improvement in speed estimation accuracy is not highly dependent on making use of the optimal value for $\beta$, and therefore, stringent calibration (and re-calibration) of $\beta$ is not likely necessary under field implementation.

\section{Conclusions and Recommendations}

The traditional practice of estimating speeds from single-loop detectors on the basis of an assumed constant average effective vehicle length generally provides results that are sufficiently inaccurate as to severely limit the usefulness of these speed estimates for real-time traffic management and traveler information.

When the traffic surveillance system contains both single-loop and dual-loop detector stations, then it is possible to directly compute average effective vehicle length at the dual-loop stations and to use this information at nearby single-loop stations. The results from this research have shown that applying this technique during each polling interval (i.e. 20-seconds) results in 
speed estimates that are significantly less accurate than those obtained using a constant average vehicle length.

If volume weighted exponential smoothing is applied to the single-loop 20-second volume and occupancy data, as well as to the calculated 20 -second average effective vehicle length from a nearby dual-loop detector station, then the speed estimates are approximately $20 \%$ more accurate than the base case. If a speed correction factor is used to adjust for systematic biases in detector calibration or vehicle population, an additional 3\% improvement is obtained. These improvements appear to be relatively insensitive to the decay coefficient $\beta$, indicating that only limited effort is likely required to calibrate the estimation method for use in practice.

The implementation of the proposed estimation method requires no new field equipment or modifications to existing field hardware. Only minor changes are needed to the software residing in the traffic management center that estimates the speed for the single-loop stations.

\section{Appendix. References}

Binkley, A. (1999) "Points of a Compass" in ITS International July/August 1999 Issue. Published by Route One Publishing Limited, United Kingdom. pages 58.

Coifman, B. (2000) "Improved velocity estimation using single loop detectors" presented at the $79^{\text {th }}$ Annual Meeting of the Transportation Research Board in Washington, DC.

Daily, D. J. (1999) "A statistical algorithm for estimating speed from single loop volume and occupancy measurements" Transportation Research Part B Vol. 33 No. 5. pages 313-322.

Hall, F. L. and Persaud B. N. (1989) "Evaluation of Speed Estimates Made from Single-Detector Data from Freeway Traffic Management Systems " in Transportation Research Record 1232, Transportation Research Board, Nation Research Council, Washington, D.C., pages 9 - 16. 
Korpal, P. R. (1992) "Incident Management: The Key to Successful Traffic Management in Toronto." ITE Journal, March 1992. Pages 58 - 61.

Pushkar, A., Hall, F. L., and Acha-Daza, J. A. (1994) "Estimation of Speeds from Single-Loop Freeway Flow and Occupancy Data Using Cusp Catastrophe Theory Model" in Transportation Research Record 1457, Transportation Research Board, Nation Research Council, Washington, D.C., pages149 - 157.

Wang, Y. and Nihan, N. L. (2000) "Freeway traffic speed estimation using single loop outputs" presented at the $79^{\text {th }}$ Annual Meeting of the Transportation Research Board in Washington, DC. 


\section{Appendix. Notation}

$D \quad=$ distance between the upstream and downstream loops in a dual-loop detector configuration $(\mathrm{km})$

$E_{n} \quad=$ average root-mean-square error for scenario $n(\mathrm{~km} / \mathrm{h})$

$I=$ number of 20-second polling intervals in 24-hour period $(I=4320)$

$K_{i} \quad=$ density computed during polling interval $i$ (vehicles/lane-km)

$L_{d} \quad=$ effective detection zone length $(\mathrm{km})$

$L_{D} \quad=$ average 24-hour effective vehicle length for dual-loop station $(\mathrm{km})$

$\bar{L}_{i} \quad=$ average effective vehicle length in polling interval $i(\mathrm{~km})$

$L_{i j} \quad=$ effective vehicle length of the $j^{\text {th }}$ vehicle in polling interval $i(\mathrm{~km})$

$L_{S} \quad=$ average 24-hour effective vehicle length for single-loop station $(\mathrm{km})$

$L_{v, i j} \quad=$ length of the $j^{\text {th }}$ vehicle in polling interval $i(\mathrm{~km})$

$L^{\prime} \quad=$ adjusted average 24-hour effective vehicle length $(\mathrm{km})$

$O_{i} \quad=$ detector occupancy for polling interval $i$

$\bar{S}^{5 h} \quad=$ estimated speed at single-loop station averaged over the time period from midnight to $5 \mathrm{AM}(\mathrm{km} / \mathrm{h})$

$\bar{S}_{d, p, i}=$ estimated space-mean-speed for polling interval $i$ of detector set $p$, on day $d(\mathrm{~km} / \mathrm{h})$

$\hat{\bar{S}}_{d, p, i}=$ observed space-mean-speed for polling interval $i$ of detector set $p$, on day $d(\mathrm{~km} / \mathrm{h})$

$\bar{S}_{D}^{5 h} \quad=$ measured speed at dual-loop station averaged over the time period from midnight to 5 $\operatorname{AM}(\mathrm{km} / \mathrm{h})$

$\bar{S}_{i} \quad=$ space-mean-speed computed for all vehicles passing the detector during polling interval $i(\mathrm{~km} / \mathrm{h})$

$T \quad=$ detector polling interval duration (hours)

$V_{i} \quad=$ equivalent hourly traffic volume for polling interval $i$ with units of vehicles per hour per lane (vphpl)

$X_{i} \quad=$ raw detector data associated with interval $i$

$Y_{i} \quad=$ smoothed detector data associated with interval $i$

$n_{i} \quad=$ number of vehicles observed to pass the detector station during polling interval $i$

$s_{i j} \quad=$ speed of the $j^{\text {th }}$ vehicle in polling interval $i(\mathrm{~km} / \mathrm{h})$ 
$t_{2, j} \quad=$ time at which the $j^{\text {th }}$ vehicle in polling interval $i$ is detected at the downstream loop of a dual-loop detector station (hours)

$t_{1, j} \quad=$ time at which the $j^{\text {th }}$ vehicle in polling interval $i$ is detected at the upstream loop of a dual-loop detector station (hours)

$\alpha_{i} \quad=$ weighting coefficient $=\beta^{n_{i}}$

$\beta=$ constant decay coefficient $(0 \leq \beta \leq 1.0)$

$\Delta_{n} \quad=$ average speed estimation accuracy improvement over the base case provided by scenario $n(\%)$

$\gamma=$ constant exponential decay coefficient $(0 \leq \gamma \leq 1.0)$ 
Table 1: Dual-Loop Detectors Stations in Detector Station Set

\begin{tabular}{|c|c|c|}
\hline Detector & \multicolumn{2}{|c|}{ Detector Station Identification } \\
Station & "Single-Loop" & "Dual-Loop" \\
\cline { 2 - 3 } Set & 401DW0110DEE & 401DW0070DEE \\
\hline 1 & 401DW0090DEC & 401DW0070DEC \\
2 & 401DW0040DEC & 401DW0020DEC \\
3 & 401DE0010DEC & 401DE0050DEC \\
4 & 401DE0050DWC & 401DE0020DWC \\
5 &
\end{tabular}


Table 2: Speed Estimation Methodologies Examined

\begin{tabular}{|c|c|c|c|}
\hline \multirow[t]{2}{*}{ Scenario } & \multicolumn{3}{|c|}{ Data Treatments } \\
\hline & $\begin{array}{l}\text { Single-Loop Volume } \\
\text { and Occupancy }\end{array}$ & $\begin{array}{l}\text { Dual-Loop Effective } \\
\text { Vehicle Length }\end{array}$ & Bias Correction \\
\hline BASE & Raw 20-sec & 24-hour Avg. & None \\
\hline 2 & Raw 20-sec & Raw 20-sec & None \\
\hline 3 & Raw 20-sec & EWMA & None \\
\hline 4 & Raw 20-sec & Vol. Weighted EWMA & None \\
\hline 5 & Vol. Weighted EWMMA & 24-hour Avg. & None \\
\hline 6 & Vol. Weighted EWMA & Raw 20-sec & None \\
\hline 7 & Vol. Weighted EWMA & EWMA & None \\
\hline 8 & Vol. Weighted EWMA & Vol. Weighted EWMA & None \\
\hline 9 & Vol. Weighted EWMA & 24-hour Avg. & Practical \\
\hline 10 & Vol. Weighted EWMA & Raw 20-sec & Practical \\
\hline 11 & Vol. Weighted EWMA & EWMA & Practical \\
\hline 12 & Vol. Weighted EWMA & Vol. Weighted EWMA & Practical \\
\hline 13 & Vol. Weighted EWMA & 24-hour Avg. & Theoretical \\
\hline 14 & Vol. Weighted EWMA & Raw 20-sec & Theoretical \\
\hline 15 & Vol. Weighted EWMA & EWMA & Theoretical \\
\hline 16 & Vol. Weighted EWMA & Vol. Weighted EWMA & Theoretical \\
\hline 17 & Raw 20-sec & 24-hour Avg. & Practical \\
\hline 18 & Raw 20-sec & Raw 20-sec & Practical \\
\hline 19 & Raw 20-sec & EWMA & Practical \\
\hline 20 & Raw 20-sec & Vol. Weighted EWMA & Practical \\
\hline \multicolumn{4}{|c|}{$\begin{array}{l}\text { EWMA = Equation (13) } \\
\text { Volume Weighted EWMA = Equation (14) } \\
\text { Practical = Equation (16) } \\
\text { Theoretical = Equation (15) } \\
\end{array}$} \\
\hline
\end{tabular}




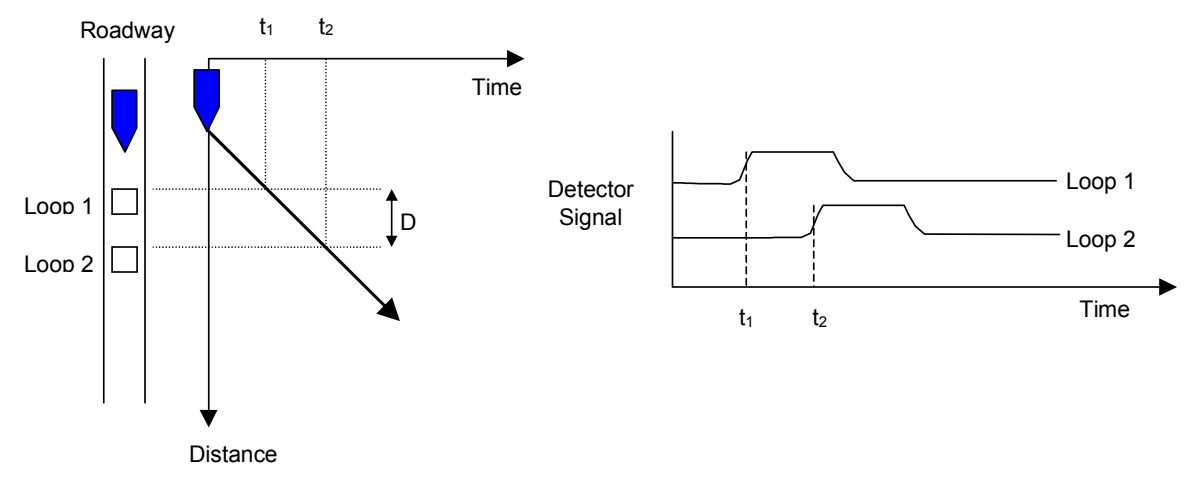

Figure 1: Time space relationship for speed measurements using dual-loop detectors 

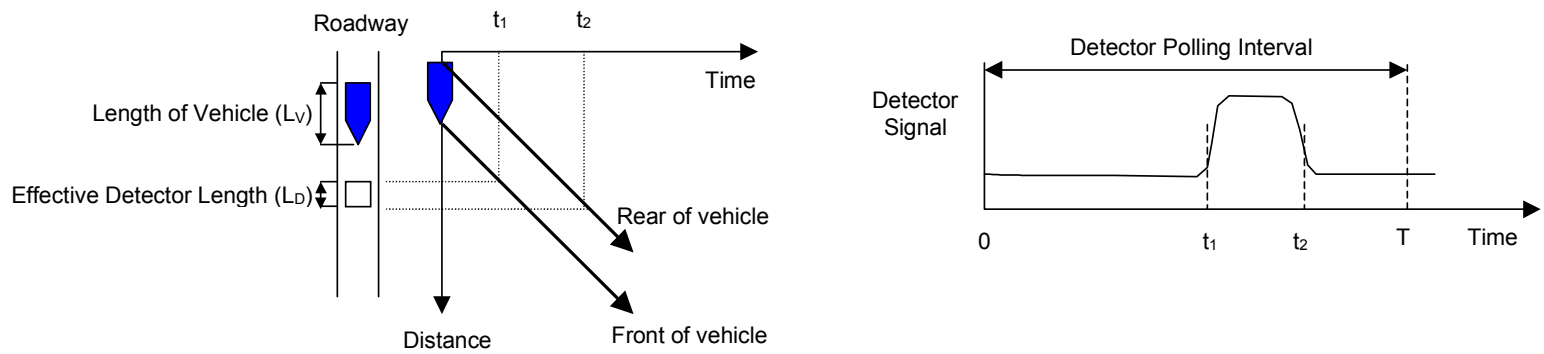

Figure 2: Time space relationship for the calculation of occupancy from single-loop detectors 


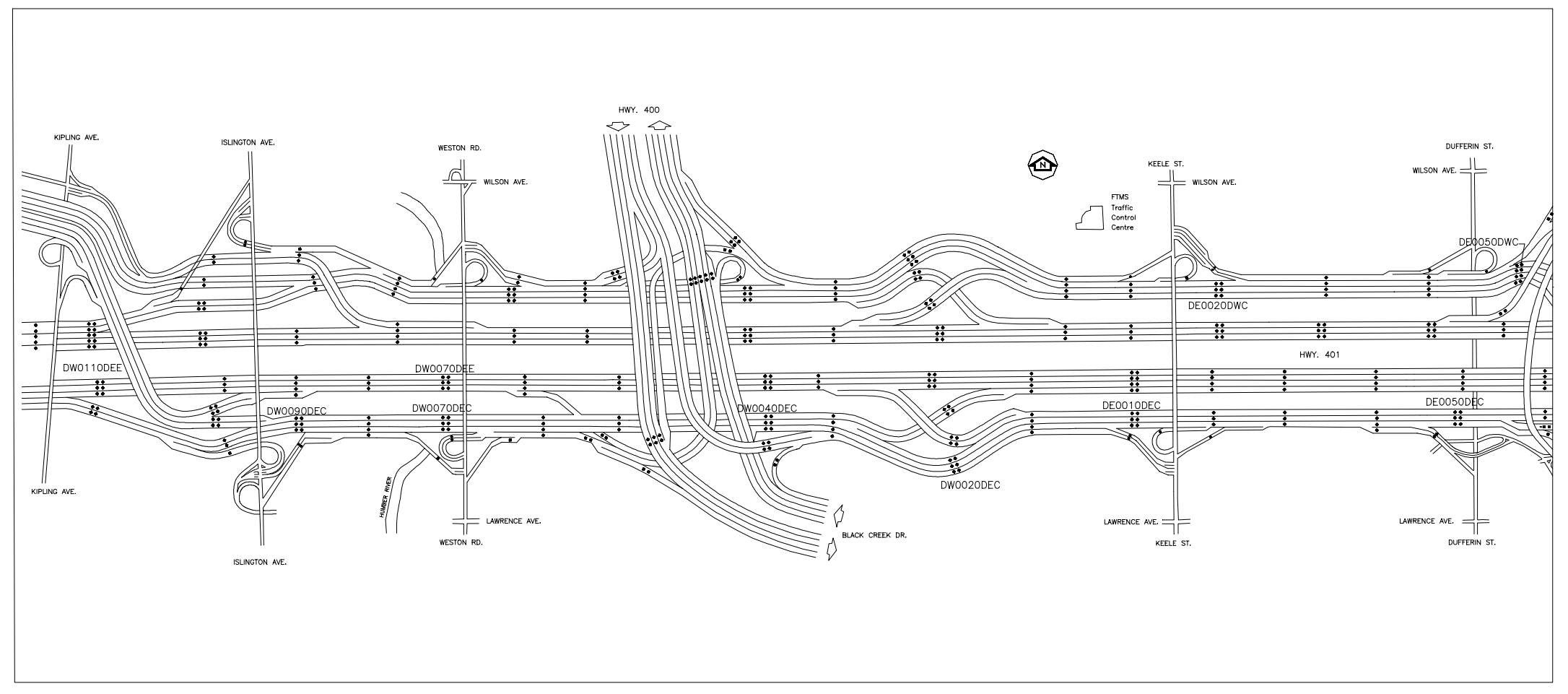

Figure 3: Location of detector stations on Highway 401 in Toronto 


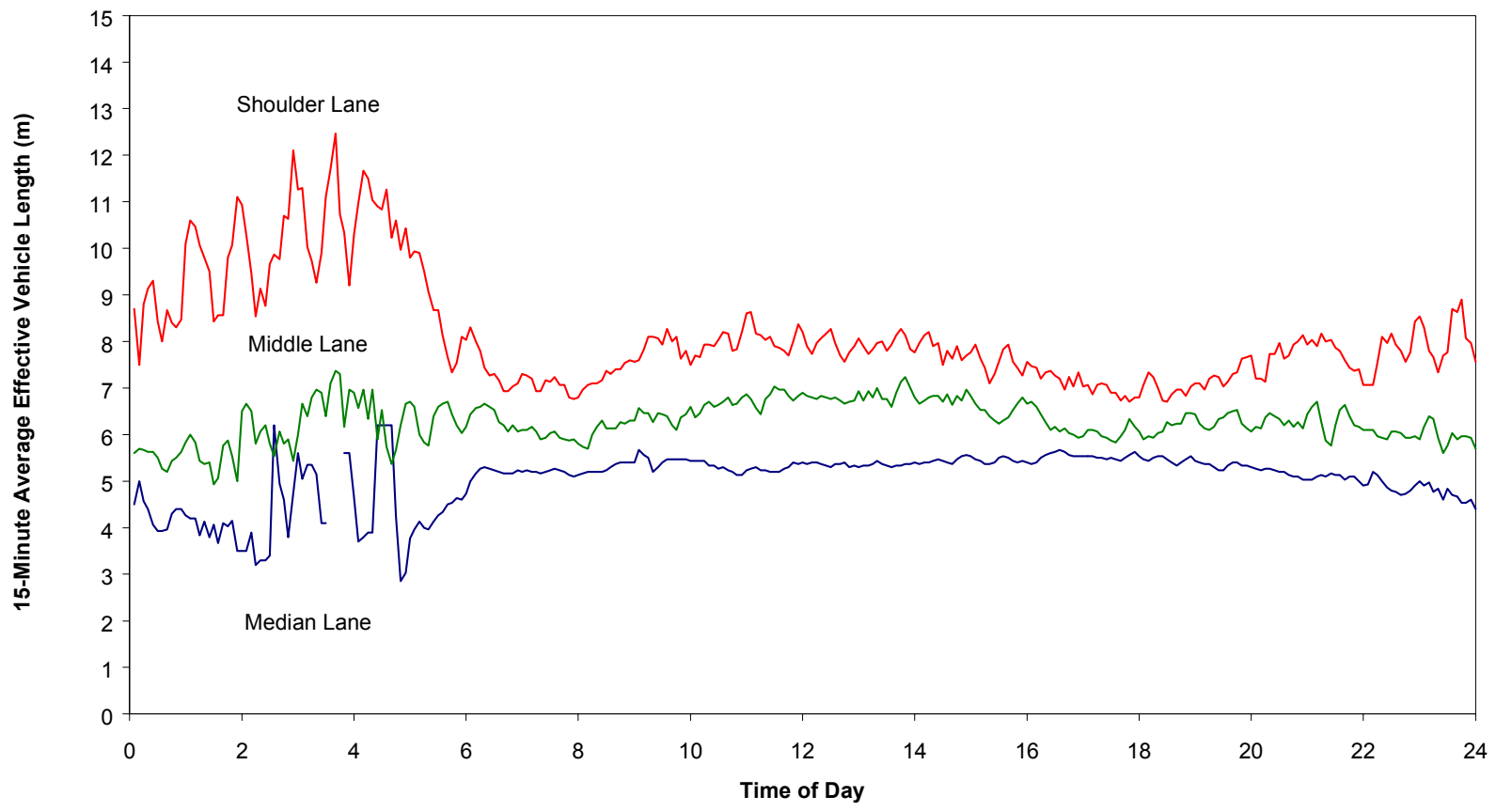

Figure 4: Temporal variation in measured 15-minute moving average effective vehicle length from Station 401DW0070DEE, October 11, 1995 


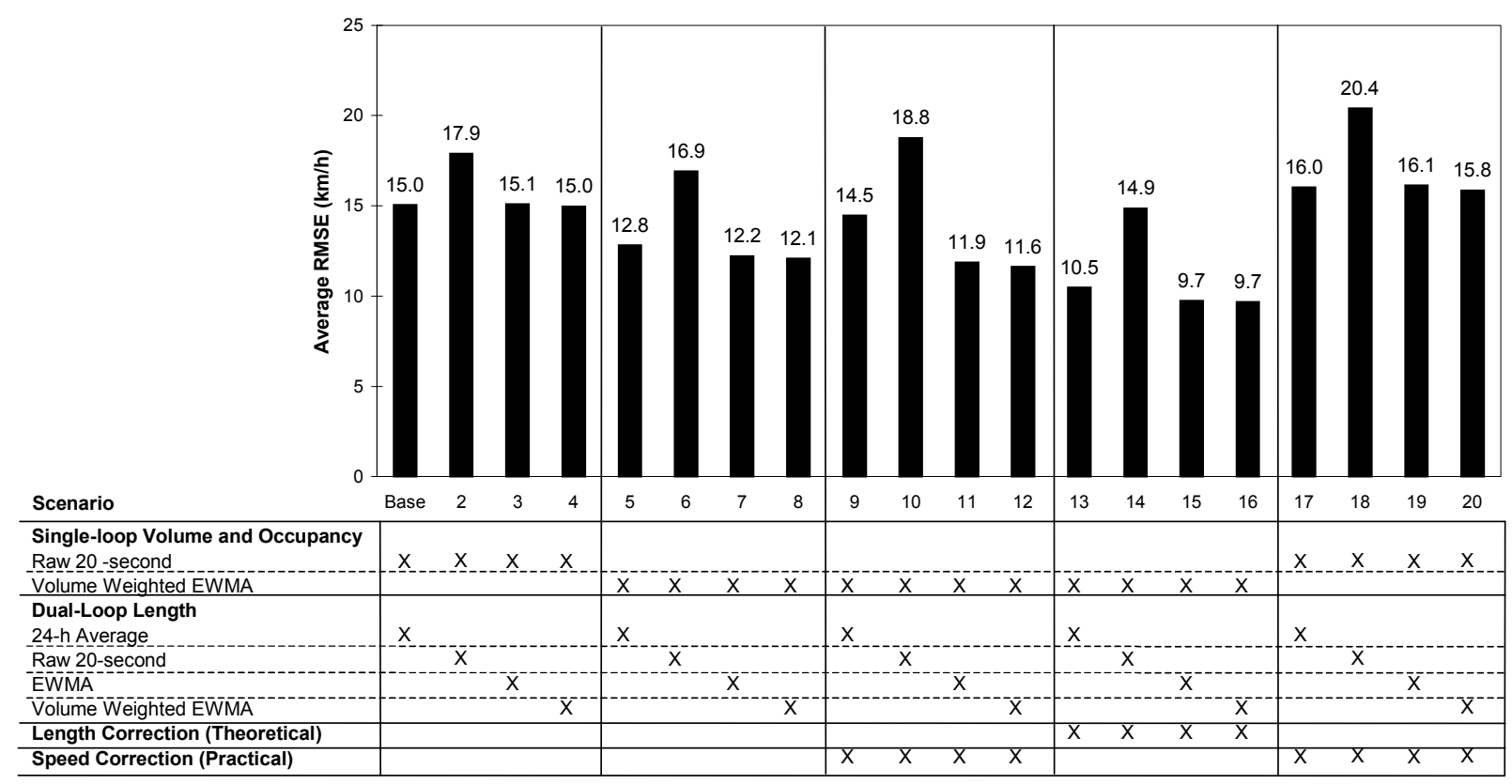

Figure 5: Average RMSE error for each speed estimation scenario 


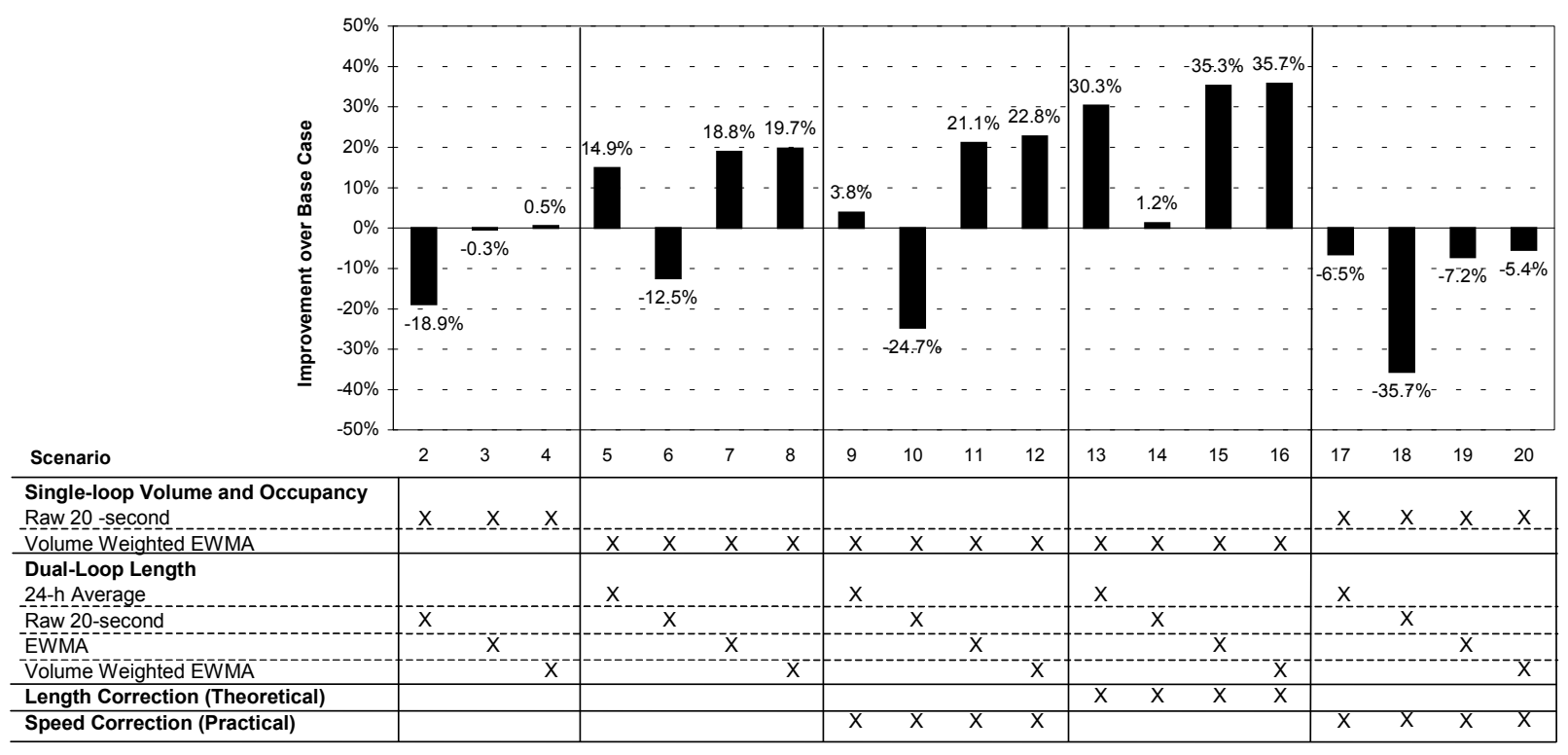

Figure 6: Improvement in speed estimates as a function of estimation scenario 


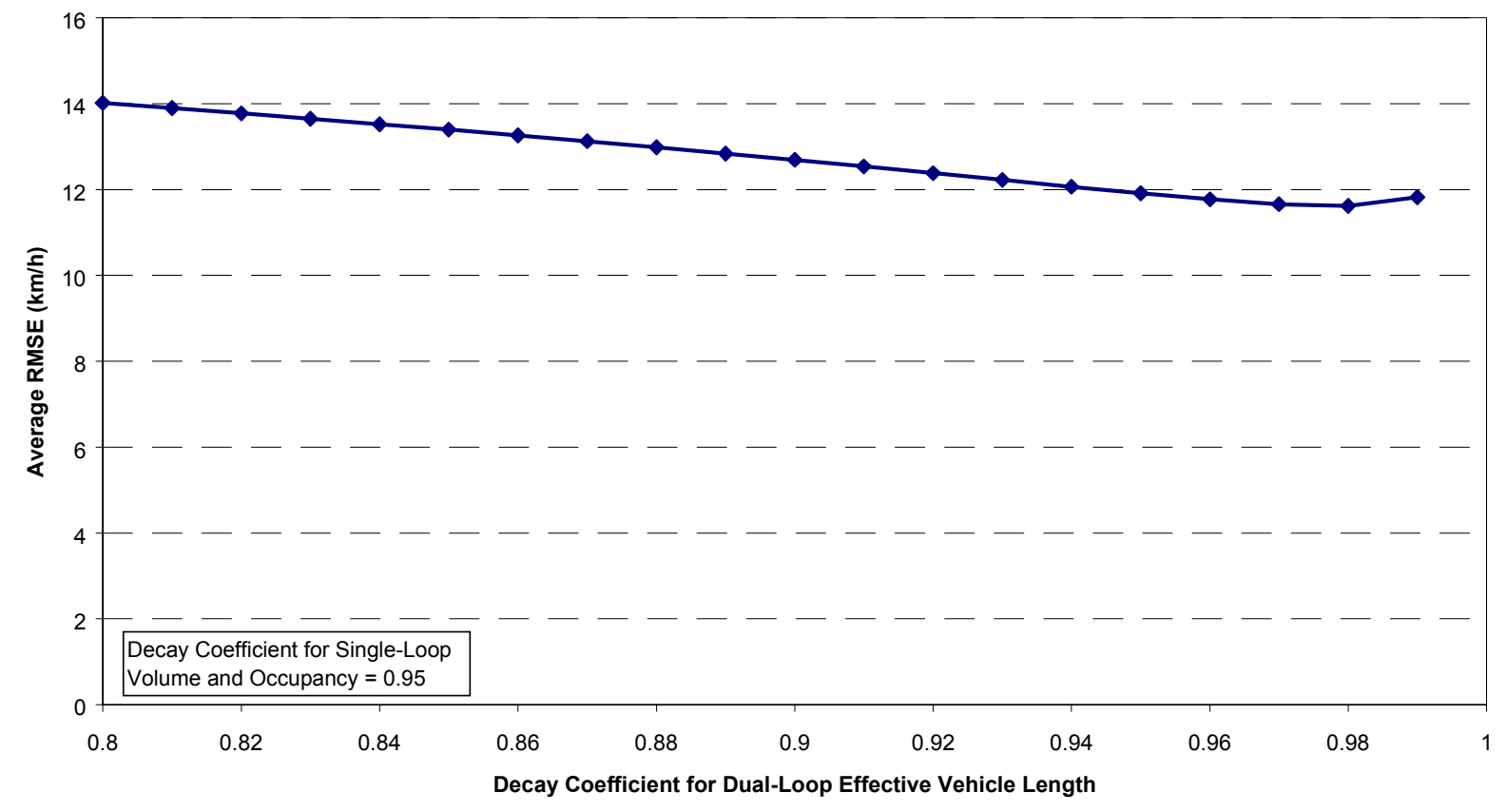

Figure 7: Sensitivity of Average RMSE as a function of decay coefficient $\beta$ for smoothing Dual-loop Effective Vehicle Length (Scenario 12) 


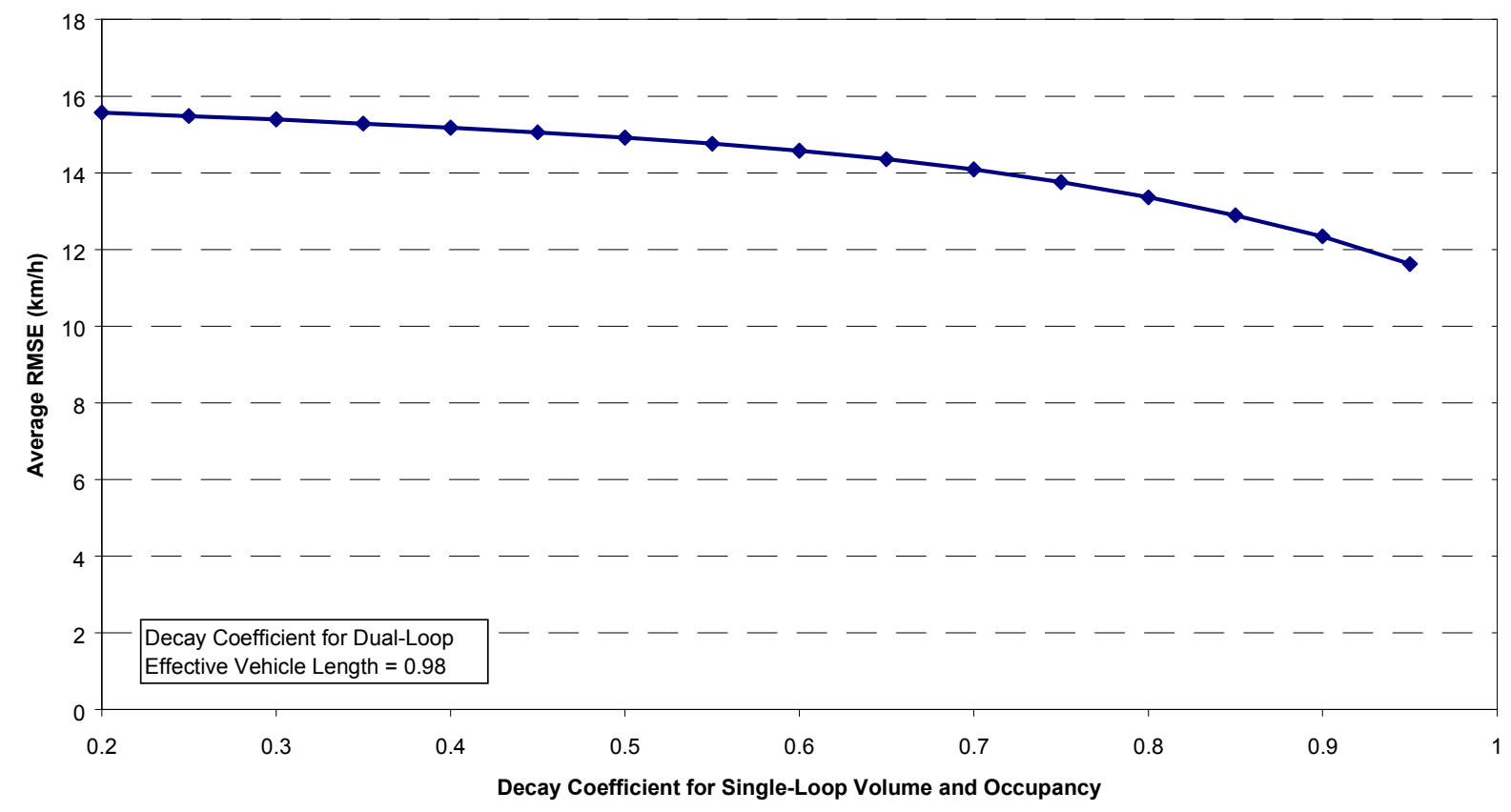

Figure 8: Sensitivity of Average RMSE as a function of decay coefficient $\beta$ for smoothing Single-loop Volume and Occupancy Measurements (Scenario 12) 